\title{
Selective Enrichment of Membrane Proteins by Partition Phase Separation for Proteomic Studies
}

\author{
M. Walid Qoronfleh,* Betsy Benton, Ray Ignacio, and Barbara Kaboord \\ Perbio Science, Bioresearch Division, 2202 N Bartlett Avenue, Milwaukee, WI 53202-1009, USA
}

Received 14 June 2002; accepted 18 December 2002

\begin{abstract}
The human proteome project will demand faster, easier, and more reliable methods to isolate and purify protein targets. Membrane proteins are the most valuable group of proteins since they are the target for $70-80 \%$ of all drugs. Perbio Science has developed a protocol for the quick, easy, and reproducible isolation of integral membrane proteins from eukaryotic cells. This procedure utilizes a proprietary formulation to facilitate cell membrane disruption in a mild, nondenaturing environment and efficiently solubilizes membrane proteins. The technique utilizes a two-phase partitioning system that enables the class separation of hydrophobic and hydrophilic proteins. A variety of protein markers were used to investigate the partitioning efficiency of the membrane protein extraction reagents (Mem-PER) (Mem-PER is a registered trademark of Pierce Biotechnology, Inc) system. These included membrane proteins with one or more transmembrane spanning domains as well as peripheral and cytosolic proteins. Based on densitometry analyses of our Western blots, we obtained excellent solubilization of membrane proteins with less than $10 \%$ contamination of the hydrophobic fraction with hydrophilic proteins. Compared to other methodologies for membrane protein solubilization that use time-consuming protocols or expensive and cumbersome instrumentation, the Mem-PER reagents system for eukaryotic membrane protein extraction offers an easy, efficient, and reproducible method to isolate membrane proteins from mammalian and yeast cells.
\end{abstract}

\section{INTRODUCTION}

Based on the sequences from several genomes, transmembrane proteins have been predicted to comprise approximately $30 \%$ of eukaryotic proteomes [1]. Membrane proteins are the most elusive and the most sought after proteins in drug discovery. They play a key role in signal transduction, cell adhesion, and ion transport and are important pharmacological targets. Yet, because of their hydrophobic and basic nature, and frequently large size, their isolation is not easy. Traditional methods for membrane isolation are often cumbersome and protein yields are poor. Techniques used for membrane protein isolation include gradient separation [2], polymer partitioning [3], and chemical treatment [4]. These methods typically result in high purity but low recovery and, with the exception of polymer partitioning, are time consuming. Detergent extraction combined with ultracentrifugation is by far the most commonly used method for membrane protein isolation $[5,6,7]$; however, this method is a multistep process involving mechanical disruption of cells followed by lengthy centrifugation prior to solubilization of the proteins in detergent.

Nonionic detergents are widely used for the solubilization and characterization of integral membrane proteins. In particular, members of the Triton $\mathrm{X}$ series are commonly employed in phase separation of these pro- teins $[6,7]$. We have developed a proprietary formulation and a protocol for the preparation of integral membrane proteins that is a nonmechanical alternative to traditional membrane protein isolation techniques. The protocol involves the gentle lysis of cells using a mild, proprietary detergent followed by membrane protein extraction utilizing the nonionic detergent, Triton X-114. Triton X-114 is a unique detergent in that it not only solubilizes membrane proteins but also separates them from hydrophilic proteins via phase partitioning at a physiological temperature [8]. Specifically, a solution of Triton $\mathrm{X}-114$ is homogeneous at $0^{\circ} \mathrm{C}$ (forms a clear micellar solution) but separates into an aqueous phase and a detergent phase above $20^{\circ} \mathrm{C}$ (the cloud point) as micellar aggregates form and the solution turns turbid. With increased temperature, phase separation proceeds until two clear phases are formed where proteins partition according to their hydrophilic and hydrophobic features. Unlike traditional protocols involving phase partitioning with Triton X-114, our protocol does not require preparation of a membrane fraction as a prerequisite for protein solubilization. Membrane proteins are extracted directly from crude cell lysates quickly and efficiently with a standard benchtop microcentrifuge. The entire procedure is completed in one hour and has been optimized for the isolation of integral membrane proteins from a variety of mammalian cell lines as well as yeast cells. 


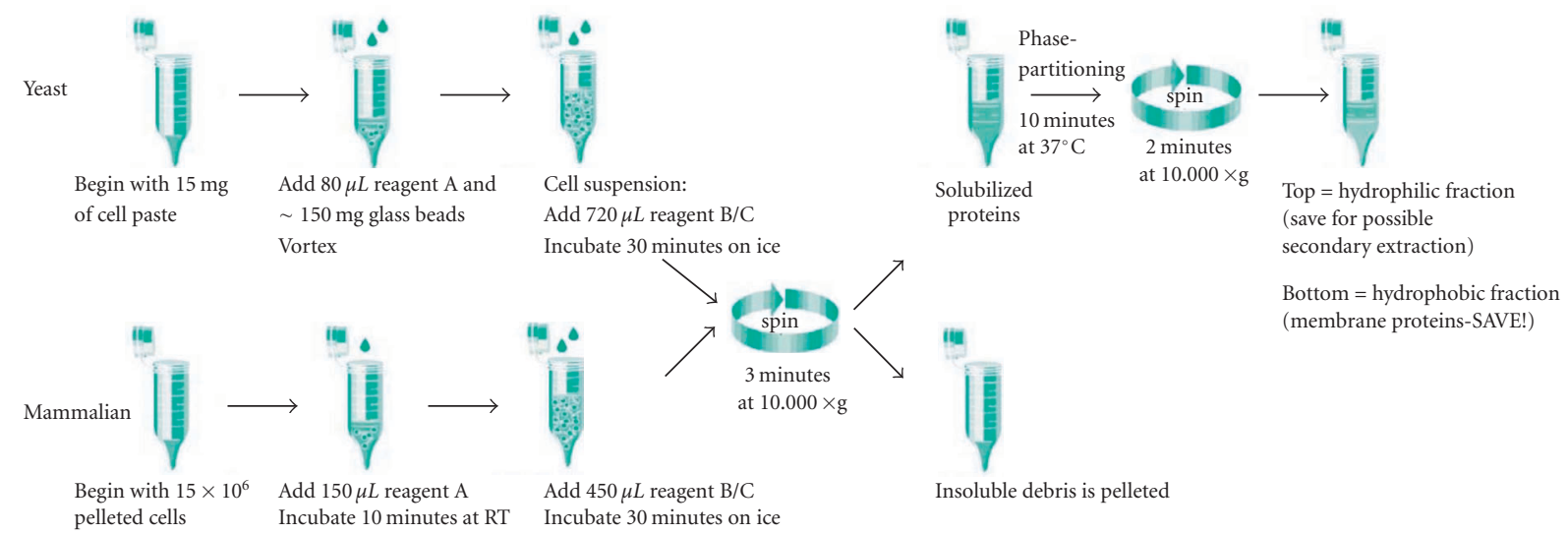

FIGURE 1. Schematic of Mem-PER Eukaryotic Membrane Protein Extraction Kit protocol. Each step of the procedure is outlined for a single extraction of either mammalian or yeast membrane proteins.

Detection and identification of proteins is facilitated through the enrichment of protein families and proteins in low abundance. Prefractionation of hydrophobic proteins enhances membrane proteomic analysis; therefore, it is essential to have reliable sample preparation methods that give high yields of this desired protein fraction. In this paper, we describe a fast, effective, and convenient protocol for membrane protein isolation involving temperature-induced phase separation of a proprietary formulation containing Triton X-114. We show that hydrophilic proteins (peripheral and cytosolic) are recovered in the aqueous phase whereas integral membrane proteins are enriched in the detergent phase. This procedure combines nonmechanical cell lysis with detergent fractionation/enrichment of membrane proteins and is termed the Mem-PER Eukaryotic Membrane Protein Extraction system.

\section{MATERIALS AND METHODS}

\section{Cell culture conditions}

Mammalian cell lines, rat brain C6, NIH-3T3, and HeLa, were obtained from American Type Culture Collection (Rockville, Md). The cells were grown to approximately $75 \%$ confluency in high-glucose Dulbecco's Modified Eagle's Medium (DMEM) supplemented with 10\% FBS, $1 \%$ antibiotic/antimycotic agent (Gibco BRL), glutamine, and sodium pyruvate. Cells were incubated at $37^{\circ} \mathrm{C}$ in an atmosphere of $5 \% \mathrm{CO}_{2}$ and harvested with $0.25 \%$ trypsin. All cell culture reagents were obtained from HyClone, Inc (Logan, Utah) except where otherwise indicated.

\section{Yeast culture conditions}

Saccharomyces cerevisiae strain EGY-194 was grown in YPD media (MobiTec, Marco Island, Fla) at $30^{\circ} \mathrm{C}$ with agitation. Cells were harvested in the exponential growth phase at a density of $1-5 \times 10^{7}$ cells $/ \mathrm{ml}$ with an $\mathrm{A}_{600}=$ $0.3-1.7$.

\section{Protein extraction protocol}

The Mem-PER system consists of three reagents. Reagent A is a cell lysis buffer, reagent B is a detergent dilution buffer, and reagent $C$ is a membrane solubilization buffer. A schematic of the protocol to extract and prepare either mammalian or yeast membrane protein fractions is shown in Figure 1. For yeast, approximately, $15 \mathrm{mg}$ of wet yeast cell paste was resuspended in MemPER reagent $A$ and vortexed with $150 \mathrm{mg}$ of $405-600 \mathrm{mi}-$ cron acid-washed glass beads for 10 minutes to disrupt the yeast cell wall. The beads were collected by pulse centrifugation, and the cell suspension was transferred to a fresh tube. Subsequent fractionation was performed according to Figure 1. Cultured NIH-3T3, HeLa, and C6 cell lines were harvested using trypsin, and were washed and pelleted in phosphate-buffered saline (PBS) at $850 \times \mathrm{g}$ for 2 minutes. Each cell pellet, containing $5 \times 10^{6}$ cells, was lysed at room temperature using Mem-PER reagent A. Yeast and mammalian membrane proteins were solubilized on ice with Mem-PER reagent $C$ diluted 2 : 1 with MemPER reagent $B$. Reagents $A$ and $B / C$ were supplemented with Halt protease inhibitors cocktail (Pierce Biotechnology, Inc, Rockford, Ill). The solubilized protein mixture was centrifuged to remove cellular debris. The clarified supernatant was heated at $37^{\circ} \mathrm{C}$ for 10 minutes followed by centrifugation to produce separate membrane and hydrophilic protein fractions. Phase partitioning resulted in the hydrophilic proteins layering at the top and the hydrophobic membrane proteins layering at the bottom. A micropipette was used to carefully remove the top (hydrophilic) phase. The hydrophobic fraction was normalized to the volume of the hydrophilic fraction using MemPER reagent $B$ diluted 4 -fold with purified water. The fractions were further diluted 2-fold with diluted MemPER reagent $B$, to decrease the detergent concentration, and boiled in $6 \mathrm{x}$-sample buffer. The isolated membrane protein fraction was used directly in SDS-PAGE and Western blotting. 


\section{Protein quantification}

The Micro BCA Protein Assay Reagent Kit (Pierce) was used to quantify extracted membrane proteins. MemPER reagent $\mathrm{C}$ was initially found to interfere with the assay because it clouds at the required incubation temperature for the assay of $37^{\circ} \mathrm{C}$; however, this interference was eliminated through dialysis using Slide-A-Lyzer MINI Dialysis Units (Pierce). Dialysis was performed overnight at $4^{\circ} \mathrm{C}$ against $25 \mathrm{mM}$ Tris, $\mathrm{pH} 7.4$, containing $0.5 \%$ CHAPS. CHAPS formed mixed micelles with reagent $\mathrm{C}$, thereby raising the cloud point of the solution above $37^{\circ} \mathrm{C}$. Approximately $100 \mu \mathrm{g}$ of total protein was obtained from $5 \times 10^{6} \mathrm{C} 6$ cells, and approximately $130 \mu \mathrm{g}$ of total membrane protein was isolated from $15 \mathrm{mg}$ of yeast cell pellet.

\section{SDS-PAGE}

Precast Novex brand (Invitrogen, Carlsbad, Calif) SDS-PAGE gels were utilized in all experiments. Standard electrophoresis conditions recommended by the gel manufacturer were employed.

\section{Optional detergent removal prior to SDS-PAGE}

Mem-PER reagent $C$ was found to interfere with electrophoresis of low molecular weight proteins. Specifically, the detergent caused lane distortion and masked protein band visualization. This was remedied by treating MemPER-isolated membrane fractions with the PAGEprep Protein Cleanup and Enrichment Kit (Pierce). The kit contains a unique resin of modified diatomaceous earth that binds protein in an organic phase of dimethyl sulfoxide (DMSO), and allows contaminating chemicals and gel-incompatible material to be washed away. Cleanup was performed according to the manufacturer's instructions.

\section{Antibodies}

Mouse monoclonal antibodies against flotillin and acetylcholinesterase (AchE) were obtained from transduction laboratories (San Diego, Calif). Heat shock protein 90 (Hsp90), a polyclonal antibody raised in goats, and cytochrome oxidase subunit 4 (Cox4), a mouse monoclonal antibody, were obtained from Santa Cruz Biotechnology, Inc (Santa Cruz, Calif). For the yeast study, proteins were detected using monoclonal antibodies obtained from Molecular Probes, Inc (Eugene, Ore). Protein bands were visualized using antigoat and antimouse secondary antibodies conjugated with horseradish peroxidase from Pierce Biotechnology, Inc.

\section{Western blotting and densitometric analysis}

Protein fractions were prepared for electrophoresis by boiling in $6 \mathrm{x}$-sample buffer. Prepared mammalian protein samples were separated using 4-20\% Tris-glycine SDSPAGE gradient gels while yeast protein samples were electrophoresed using NuPAGE 4-12\% Bis-Tris gels. The protein fractions were then blotted to nitrocellulose. The blots were blocked in Superblock blocking buffer (Pierce) containing $0.05 \%$ Tween-20. After probing with primary and secondary antibodies, detection was performed with SuperSignal West Femto Maximum Sensitivity Substrate (Pierce) for 5 minutes followed by exposure to X-ray film for 15 seconds or to a FluorChem CCD camera (Alpha Innotech Corp, San Leandro, Calif) for 2 minutes. Bands were quantified using densitometry analysis (AlphaEaseFC software, Alpha Innotech) and expressed as a percentage of the total protein in the combined hydrophilic and hydrophobic (membrane protein) fractions.

\section{RESULTS}

\section{Protein fractionation protocol}

The membrane protein extraction protocol was accomplished in two parts (Figure 1). First, cells were lysed with a proprietary detergent and then a second proprietary formulation containing Triton X-114 was added to solubilize the membrane proteins. A white, flocculent material appeared following addition of the cell lysis component, Mem-PER reagent A. This debris was likely comprised of lipid and cell membrane material but not DNA since the addition of DNase was not found to diminish the particulate. Solubilization of the membrane proteins with Mem-PER reagent $C$ diluted with Mem-PER reagent $B$ was performed on ice with vortexing every 5 minutes. Longer incubation was not found to increase extraction efficiencies (data not shown). The cellular debris was removed during subsequent centrifugation. The hydrophobic proteins were then separated from the hydrophilic proteins through phase partitioning $[8]$ at $37^{\circ} \mathrm{C}$. Following careful separation of the two layers with a micropipette, membrane proteins were ready for subsequent analysis. Complete separation of the two layers was not possible due to the transient nature of the interface. No more than 10 samples were processed at one time since the interface slowly disappeared as the temperature of the sample fell below $37^{\circ} \mathrm{C}$. Although a distinct separation could be seen, a small amount of crossover of each phase into the other could not be avoided during pipetting. In order to minimize contamination of the hydrophobic layer with the hydrophilic layer, some of the hydrophobic layer was sacrificed during removal of the top layer. A second round of extraction of the hydrophilic fraction obtained was not found to significantly increase membrane protein yields (data not shown). The hydrophilic, hydrophobic, and insoluble debris fractions were analyzed by SDS-PAGE and Western blotting.

\section{Membrane protein extraction from mammalian cells}

Mem-PER reagents were found to be highly efficient in the extraction of integral membrane proteins containing one or two transmembrane spanning domains. These results were found to be consistent with three different 


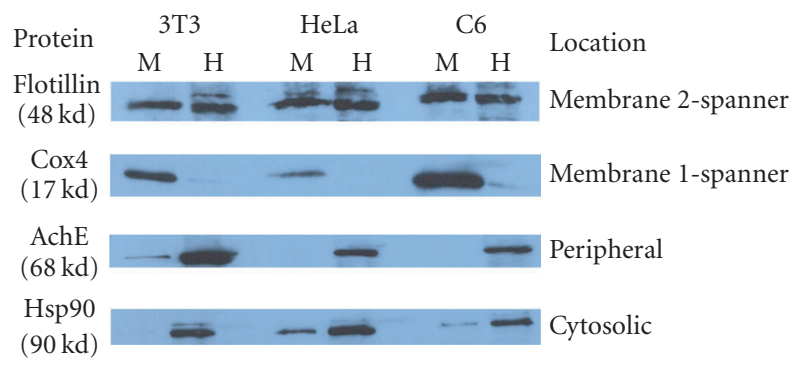

Figure 2. Partitioning of solubilized mammalian membrane proteins using the Mem-PER Kit. Proteins from three cell lines were solubilized and extracted using the Mem-PER Kit. Each set of hydrophilic and hydrophobic (membrane protein) fractions obtained was normalized to one another and analyzed by Western blot for four proteins from the cellular locations noted. PAGEprep resin was used to remove the detergent from the membrane fraction prior to SDS-PAGE/Western analysis of Cox4 due to the interference of detergent with band migration of low molecular weight proteins. A negligible amount of protein was found in all debris fractions (data not shown). Abbreviations: Acetylcholinesterase (AchE), cytochrome oxidase subunit 4 (Cox4), heat shock protein 90 (Hsp90), $\mathrm{M}=$ solubilized membrane protein fraction, $\mathrm{H}=$ hydrophilic protein fraction.

TABLe 1. Quantification of mammalian cell lysate proteins fractionated with the Mem-PER reagents in Figure 2.

\begin{tabular}{cccccc}
\hline Cell type & Fraction* & Flotillin (2-spanner) & Cox4 (1-spanner) & AchE (Peripheral) & Hsp90 (Cytosolic) \\
\hline \multirow{2}{*}{ NIH-3T3 } & Membrane & 45.0 & 90.8 & 1.7 & 6.8 \\
& Hydrophilic & 55.0 & 9.2 & 89.3 & 93.2 \\
\hline \multirow{2}{*}{ HeLa } & Membrane & 48.4 & 10.8 & 95.9 & 15.5 \\
& Hydrophilic & 51.6 & 94.5 & 6.4 & 84.4 \\
\hline \multirow{2}{*}{ C6 } & Membrane & 56.0 & 5.5 & 93.6 & 10.6 \\
& Hydrophilic & 44.0 & & 89.4 \\
\hline
\end{tabular}

* Percent recovery of proteins following extraction is expressed as a percentage of the total protein in the combined hydrophilic and hydrophobic (membrane protein) fractions. The data was obtained from a single experiment but is representative of results obtained in multiple independent experiments.

mammalian cell lines, C6, NIH-3T3, and HeLa. As shown in Figure 2 and Table 1, the integral plasma membrane protein flotillin, containing two transmembrane domains, was extracted with an efficiency of approximately 50\% from the three cell lines. These reported values were found to be reproducible in several isolated experiments. Extraction of Cox4, an outer mitochondrial membrane protein containing one transmembrane domain, was found to be even more efficient with approximately $90 \%$ recovery in the hydrophobic fractions obtained from the three cell lines. The membrane fraction probed for Cox 4 $(17 \mathrm{kd})$ in Figure 2 was treated with the PAGEprep resin prior to electrophoresis. The detergent in Mem-PER reagent $\mathrm{C}$ was found to interfere with electrophoresis of low molecular weight proteins (Figure 3 ) but did not affect electrophoresis of mid to high molecular weight proteins.

Cross-contamination of cytosolic and peripheral proteins into the prepared hydrophobic fraction was minimal. AchE, a peripheral protein, and Hsp90, a cytosolic protein, were routinely found to partition into the hydrophilic fraction with an efficiency of $>90 \%$. The remaining $10 \%$ or less found in the hydrophobic fraction was likely due in part to difficulty in obtaining complete

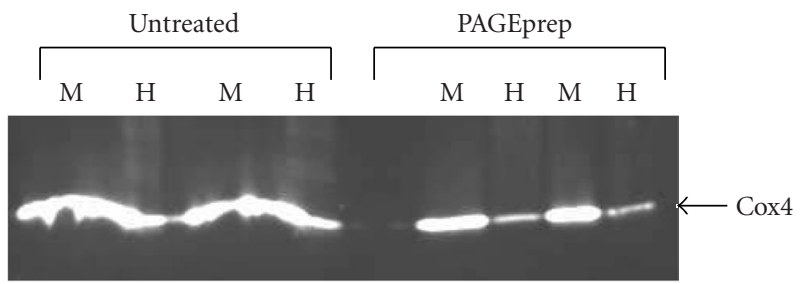

FIGURE 3. Removal of detergent from cell lysis fractions by the PAGEprep resin. Rat C6 cells were lysed and a membrane protein fraction isolated using the Mem-PER reagents. Membrane (M) and hydrophilic (H) cell fractions were separated by SDSPAGE (4-20\% gradient gel) with or without prior treatment using PAGEprep resin to remove detergent. Western blot analysis was performed as described in materials and methods using an antibody against Cox4. PAGEprep-treated samples show better band resolution than samples that were untreated and still contained the detergent.

separation at the interface between the two phases, and the slow disappearance of the interface over time when the temperature fell below the cloud point of the mixture. Insoluble debris pellets typically contained $<5 \%$ of the membrane proteins examined in this study. 


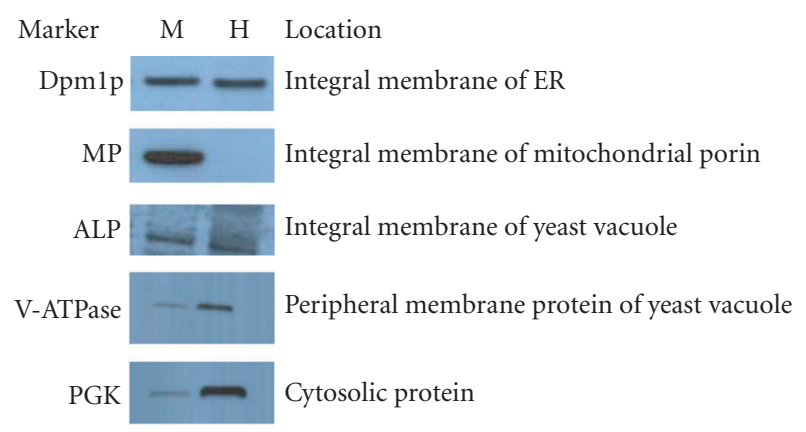

FIGURE 4. Partitioning of solubilized yeast membrane proteins using the Mem-PER Kit. Yeast proteins from $S$ cerevisiae (strain: EGY194) were solubilized and partitioned based on hydrophobic phase separation. Several proteins were solubilized and extracted using the Mem-PER reagents. Partitioning efficiency was determined through Western blot of normalized samples. A negligible amount of protein was found in all debris fractions (data not shown). Abbreviations: mitochondrial porin (MP), 3-phosphoglycerate kinase (PGK), alkaline phosphatase (AP), Dol-P-Man synthase (Dpm1p), $\mathrm{M}=$ solubilized membrane protein, $\mathrm{H}=$ hydrophilic protein fraction.

TABLE 2. Quantification of yeast proteins fractionated with the Mem-PER reagents in Figure 4.

\begin{tabular}{cccccc}
\hline Fraction* & Dpmlp & MP & AP & V-ATPase & PGK \\
\hline Membrane & 52.4 & 98.4 & 57.2 & 22.1 & 9.1 \\
Hydrophilic & 47.6 & 1.6 & 42.3 & 77.9 & 90.9 \\
\hline
\end{tabular}

* Percent recovery of proteins following extraction is expressed as a percentage of the total protein in the combined hydrophilic and hydrophobic (membrane protein) fractions. The data was obtained from a single experiment but is representative of results obtained in three independent experiments.

\section{Membrane protein preparation from yeast}

Extraction efficiencies seen with the mammalian cell lines were similar to those obtained with yeast cells. Glass beads were used to lyse the rigid cell wall, and preparation of the membrane proteins was then performed with the Mem-PER reagents according to the same protocol used for the mammalian cells. Several protein markers were used to monitor the efficiency of the Mem-PER system to solubilize and isolate yeast integral membrane proteins. Figure 4 and Table 2 show the partitioning efficiency observed for these proteins. Mitochondrial porin (MP), an integral membrane protein of the outer mitochondrial membrane containing one transmembrane domain, was extracted into the hydrophobic fraction with an efficiency of greater than $90 \%$ with very negligible cross-contamination from the hydrophilic fraction. PGK (3-phosphoglycerate kinase), a cytosolic protein, was extracted into the hydrophilic fraction with an efficiency of greater than $85 \%$. Alkaline phosphatase (ALP), an integral membrane protein of yeast vacuoles, was extracted into the hydrophobic fraction with an efficiency of $>$ $50 \%$ whereas greater than $70 \%$ of V-ATPase, a peripheral membrane protein of yeast vacuoles, was recovered in the hydrophilic fraction. Dol-P-Man synthase (Dpm1p), a membrane protein in the yeast endoplasmic reticulum containing one transmembrane spanning domain, was extracted into the hydrophobic fraction with an efficiency of $50 \%$. Similar results were obtained for all of these proteins in several isolated experiments. Insoluble debris pellets typically contained between 3 and $20 \%$ of the yeast membrane proteins examined in this study.

\section{DISCUSSION}

Transmembrane proteins are a valuable family of proteins. Functionally they are central to cell life and are the target of about $80 \%$ of all drugs. Preparation of membrane proteins is time consuming and difficult; therefore, development of analytical systems that allow the isolation and identification of this group of proteins would be desirable. Ideally, the isolation process should be mild yet rapid. Detergents have played significant roles in this effort $[6,7]$. Detergents serve as invaluable tools to isolate, solubilize, and manipulate membrane proteins for subsequent biochemical and biophysical characterization [9]. Consequently, our understanding of the structure and function of membrane proteins has advanced significantly over the past decade. Nonionic detergents have been useful in this regard since they are widely used for the solubilization and characterization of integral membrane proteins. These proteins can be separated from hydrophilic proteins using the nonionic detergent Triton X-114 that undergoes separation at physiological temperatures into detergent-rich and aqueous-rich phases [8]. 
Many fractionation protocols exist for the enrichment of hydrophobic proteins $[4,6]$; however, isolation of these proteins can be a tedious and time-consuming process requiring gradient methods and expensive ultracentrifugation equipment. A more convenient fractionation of membrane proteins can be achieved through the use of detergents. We have developed a proprietary mild detergent formulation and a protocol for the lysis of cells followed by enrichment of hydrophobic proteins via phase separation. Our unique cocktail contains Triton X-114. The protocol was performed with a benchtop microcentrifuge and did not require mechanical lysis such as sonication or Dounce homogenization. Separations were performed on a microscale; however, similar methodology using phase partitioning has been demonstrated on a large preparative scale [10]. We obtained membrane protein extracts quickly and efficiently from mammalian cells. Yeast membrane proteins were obtained in a similar fashion, except that the yeast cell wall was first removed. Glass beads were found to disrupt the cell wall quickly and efficiently [11] and were effective when used in combination with the cell lysis reagent, Mem-PER reagent A. Extraction efficiencies of approximately $50 \%$ or greater were typically seen with proteins containing one or two transmembrane spanning domains. Lower yields may be obtained with more complex integral membrane proteins, and variability in extraction efficiency may be observed depending on factors such as posttranslational modifications, and the number of transmembrane spanning domains. In addition, anomalous partitioning of some integral membrane glycoproteins has been observed. For example, intact acetylcholine receptor, an integral membrane protein containing four transmembrane domains, has been shown to partition into the aqueous-rich hydrophilic phase [12]. The reason for this behavior may be due to the large hydrophilic moieties on the glycoprotein and/or the channel-forming property of this protein. Membrane protein activity may be maintained following separation into the mild detergent environment of the Mem-PER system. Several integral membrane proteins have been found to retain their biological activity when solubilized in nonionic detergents [13]; however, retention of activity is dependent on the characteristics of the protein being analyzed and cannot be assured.

Triton X-114 is an effective reagent for the isolation of membrane proteins from mammalian systems. This method of fractionation has been used to isolate $75 \%$ of the integral membrane glycoproteins from prepared erythrocyte membranes [8] and nearly $100 \%$ of cytochrome b558 from prepared bovine granulocytes [14]. It has also been used to solubilize membrane proteins of subcellular fractions from the bovine adrenal medulla [15] as well as hepatic Golgi membrane proteins [16]. In all of these reports, an initial purification was performed prior to phase partitioning in Triton X-114. Our protocol was designed for crude cell lysates and does not require prior processing.
Membrane protein extracts obtained using Triton $\mathrm{X}-114$ have been used in many downstream applications. Golgi proteins partitioned with the nonionic detergent were analyzed by mass spectrometry following onedimensional SDS-PAGE [16]. Hydrophobic proteins isolated using Triton X-114 have also been analyzed by 2D gel electrophoresis following removal of the detergent using hydroxyapatite column chromatography [17]. A combination of 2D electrophoresis and mass spectrometry was used to identify a hydrophobic receptor protein, verylow-density lipoprotein, in the detergent-enriched phase and the cytoplasmic protein Hsp90 in the aqueous phase. Clearly, phase separation of Triton X-114 is a useful tool in the prefractionation of membrane proteins, and the detergent-rich extracts obtained with this method have been used successfully in proteomics applications.

The Mem-PER system is an excellent tool for the initial purification and preparation of protein fractions for downstream analysis. It provides a rapid and convenient protocol for the reproducible partitioning of mammalian and yeast proteins into hydrophobic and hydrophilic fractions. Interestingly, the extraction of yeast membrane proteins has never been performed with phase partitioning, and to our knowledge this is the first report. Prefractionation of complex protein mixtures is critical for proteomic studies because it increases the resolving power of many analytical techniques by allowing for the identification of low-abundance proteins. Selective separation of hydrophobic proteins enhances membrane proteomic examination. Integral membrane proteins cannot be extracted easily; however, phase partitioning is a proven and valuable technique for the enrichment of this important protein family.

\section{REFERENCES}

[1] Wallin E, von Heijne G. Genome-wide analysis of integral membrane proteins from eubacterial, archaean, and eukaryotic organisms. Protein Sci. 1998;7(4):1029-1038.

[2] Morre DJ. Isolation and purification of organelles and endomembrane components from rat liver. In: Chrispeels MJ, ed. Molecular Techniques and Approaches in Developmental Biology. New York, NY: John Wiley;1973:1-27.

[3] Morre DJ, Morre DM. Preparation of mammalian plasma membranes by aqueous two-phase partitioning. Biotechniques. 1989;7(9):946-958.

[4] Lenstra JA, Bloemendal H. Topography of the total protein population from cultured cells upon fractionation by chemical extractions. Eur J Biochem. 1983;135(3):413-423.

[5] Ohlendieck K. Extraction of membrane proteins. In: Protein Purification Protocols. Methods in Molecular Biology. vol. 59. Totowa, NJ: Humana Press Inc;1996:293-304.

[6] Helenius A, Simons K. Solubilization of membranes 
by detergents. Biochim Biophys Acta. 1975;415(1): 29-79.

[7] Tanford C, Reynolds JA. Characterization of membrane proteins in detergent solutions. Biochim Biophys Acta. 1976;457(2):133-170.

[8] Bordier C. Phase separation of integral membrane proteins in Triton X-114 solution. J Biol Chem. 1981;256(4):1604-1607.

[9] Garavito RM, Ferguson-Miller S. Detergents as tools in membrane biochemistry. J Biol Chem. 2001;276(35):32403-32406.

[10] Pryde JG. Triton X-114: a detergent that has come in from the cold. Trends Biochem Sci. 1986;11:160-163.

[11] Panaretou B, Piper P. Isolation of yeast plasma membranes. In: Evans IH, ed. Yeast Protocols: Methods in Molecular Biology. vol. 53. Totowa, NJ: Humana Press Inc;1996:117-121.

[12] Maher PA, Singer SJ. Anomalous interaction of the acetylcholine receptor protein with the nonionic detergent Triton X-114. Proc Natl Acad Sci U S A. 1985;82(4):958-962.

[13] Tzagoloff A, Penefsky HS. Extraction and purification of lipoprotein complexes from membranes. Methods Enzymol. 1971;XXII:204-219.

[14] Pember SO, Heyl BL, Kinkade JM Jr, Lambeth JD. Cytochrome b558 from (bovine) granulocytes. Partial purification from Triton X-114 extracts and properties of the isolated cytochrome. J Biol Chem. 1984;259(16):10590-10595.

[15] Pryde JG, Phillips JH. Fractionation of membrane proteins by temperature-induced phase separation in Triton X-114. Application to subcellular fractions of the adrenal medulla. Biochem J. 1986;233(2):525533.

[16] Bell AW, Ward MA, Blackstock WP, et al. Proteomics characterization of abundant Golgi membrane proteins. J Biol Chem. 2001;276(7):5152-5165.

[17] Wissing J, Heim S, Flohe L, Bilitewski U, Frank R. Enrichment of hydrophobic proteins via Triton $\mathrm{X}-114$ phase partitioning and hydroxyapatite column chromatography for mass spectrometry. Electrophoresis. 2000;21(13):2589-2593.

\footnotetext{
* Corresponding author. E-mail: walid.qoronfleh@perbio.com Fax: +1 414227 3759; Tel: +1 4142273605
} 

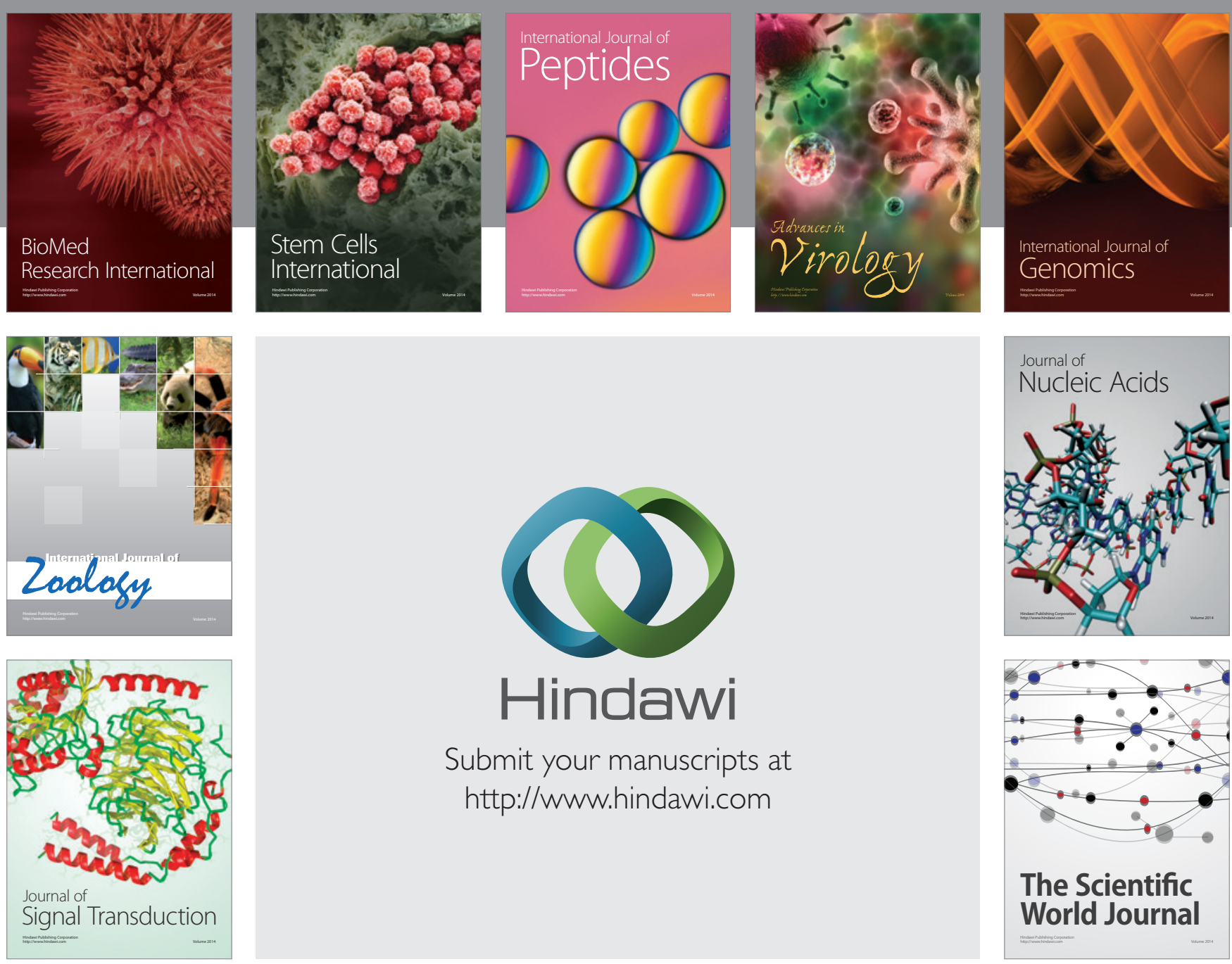

Submit your manuscripts at

http://www.hindawi.com
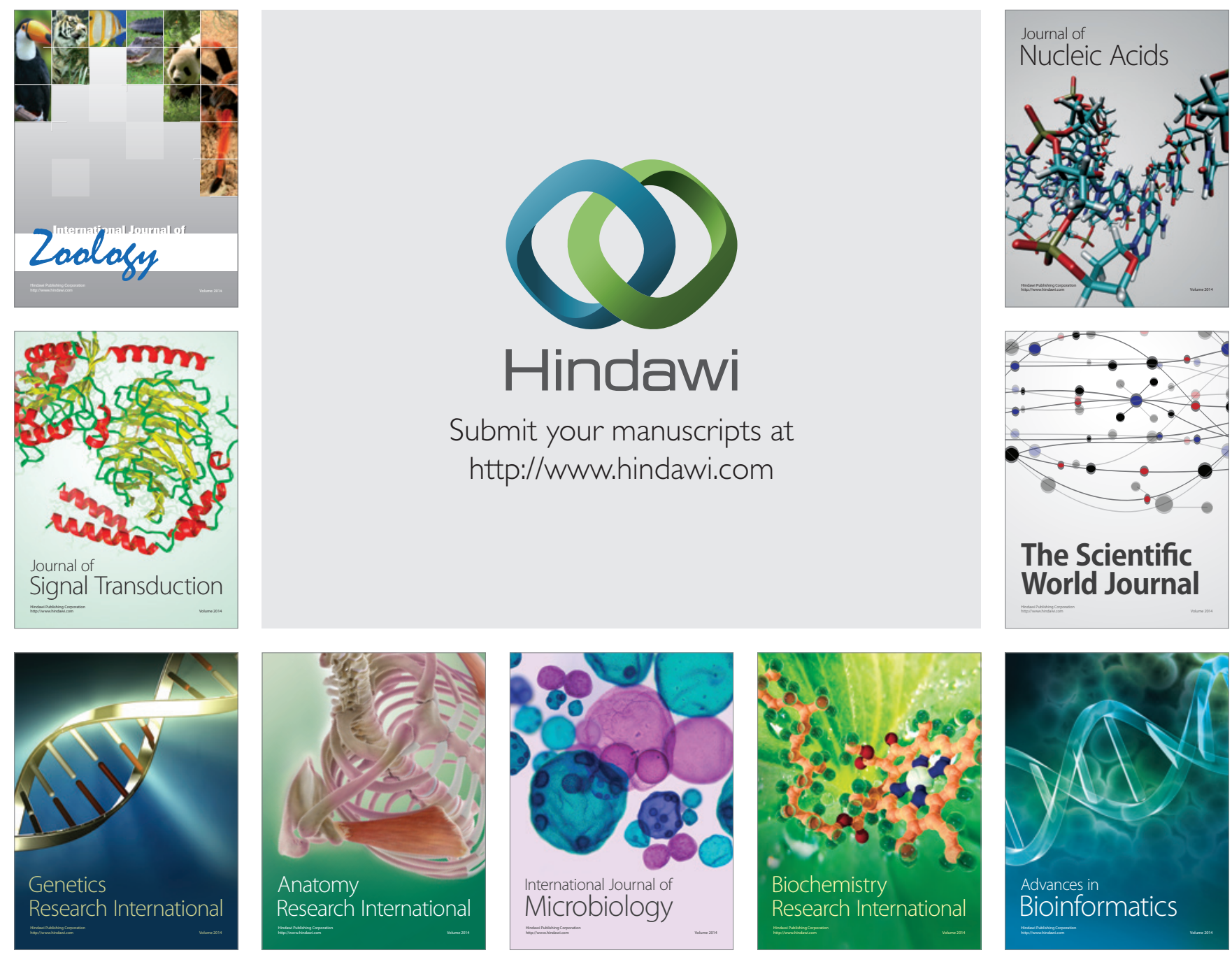

The Scientific World Journal
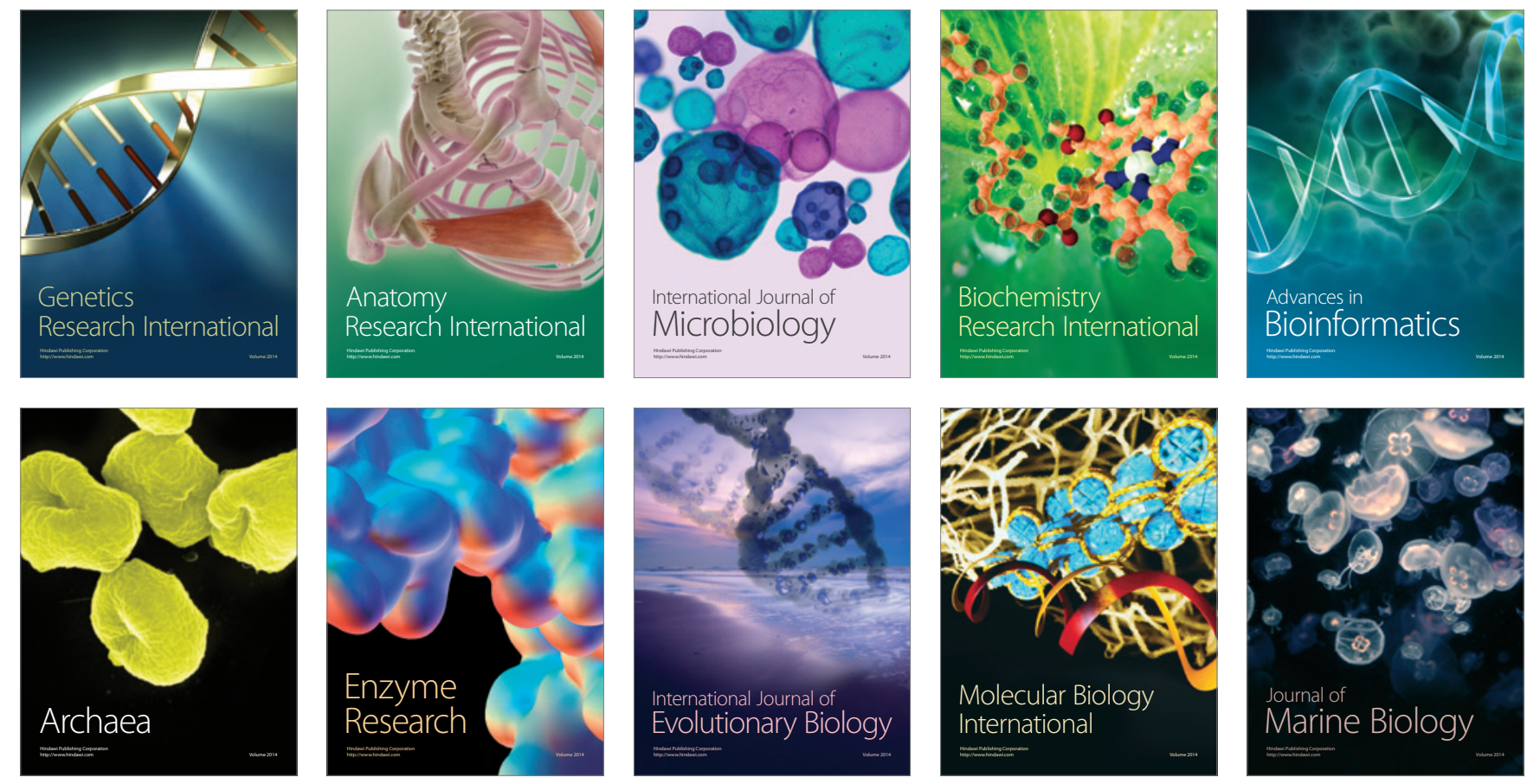